\title{
Solitary Endobronchial Papilloma: A Rare Entity
}

\author{
Muhammad Soban Arif Maan1, Muhammad Waleed ${ }^{1}$, Amir Humza Sohail1, Asna Sulaiman' ${ }^{1}$ and Javaid Ahmed Khan² \\ ${ }^{1}$ Medical College, The Aga Khan University, Karachi, Pakistan \\ 2Department of Pulmonology and Critical Care, The Aga Khan University, Karachi, Pakistan
}

\begin{abstract}
Solitary papillomas of the lower airways are uncommon clinical entities that present a diagnostic challenge because they are not usually considered among the differential diagnoses. Reliable clinical data about their presentation and treatment remain scarce. We describe a case of an elderly male who presented to us with cough and hemoptysis. He had been a smoker for 50 years. Chest radiograph revealed hyperinflated lungs and slight hilar enlargement on the right side. Bronchoscopy revealed a polypoid mass at the origin of the right upper lobe bronchus. Biopsy showed it to be a papillomatous growth composed of squamous epithelial cells. No malignant cells were detected microscopically. Patient declined surgical removal of this papilloma. He was fine on followup visits during the subsequent months. In patients with hemoptysis, solitary endobronchial papilloma should also be considered in the differential diagnosis
\end{abstract}

Key Words: Papilloma, Endobronchial, Hemoptysis.

How to cite this article: Maan MSA, Waleed M, Sohail AH, Sulaiman A, Khan JA. Solitary endobronchial papilloma: A rare entity. J Coll Physicians Surg Pak 2019; 29 (Supplement 2):S120-S122.

\section{INTRODUCTION}

Solitary papillomas of the lower airways, also known as solitary endobronchial papillomas (SEPs), are benign tumours that are rarely encountered in the clinic. The etiology of these neoplasms is not fully understood, although smoking and human papilloma virus (HPV) infection are hypothesised to have a contributory role. 1 SEPs have been divided for ease of management into three histologically distinct sub-categories. ${ }^{1}$ Treatment for SEPs remains a grey area primarily because of the dearth of clinical data available due to their rarity of this pathology. Herein, we present the case of a 73-year male who presented to us with cough and hemoptysis and was subsequently found to have a solitary squamous papilloma located in the right bronchus. We will also briefly review briefly the main diagnostic and treatment modalities that have proven effective for SEPs according to the existing literature.

\section{CASE REPORT}

A 73-year male presented to the emergency room with complaints of cough and hemoptysis for three days. The hemoptysis was fresh blood, about a quarter cup, roughly four to five times a day with no clots. The patient had been a smoker for the past 50 years and had consistently smoked 3 to 4 packs of cigarettes per day.

Correspondence to: Dr. Muhammad Soban Arif Maan,

Medical College, The Aga Khan University, Stadium Road,

Karachi, Pakistan

E-mail: sobanmaan@live.co.uk

Received: December 14, 2018; Revised: April 08, 2019;

Accepted: June 13, 2019
He was a tobacco chewer as well. He had been using homeopathic drugs for several years, along with several multivitamin supplements and antibiotics prescribed by various general practitioners. He was a known case of chronic obstructive pulmonary disease (COPD). Clinically, he looked well, slightly overweight and afebrile. On examination, the chest expansion was decreased on both sides, and the percussion note was noticeably hyper-resonant on the right side. Right mid zone crackles were present and the anteroposterior (AP) diameter was increased. There was normal vesicular breathing with minimal wheezes bilaterally. His pulse was regular. Rest of the general physical examination was unremarkable.

His routine investigations (including complete blood count, urea, creatinine and electrolyte levels) were within normal limits. Chest X-ray showed hyperinflated lungs with a prominent right hilum.

The patient was managed as a case of exacerbated COPD and admitted for further workup. A bronchoscopy was done. The bronchoscopy revealed normal vocal cords with some overlying blood which was aspirated. The right bronchial tree was normal except at the origin of the right upper lobe bronchus, where a polypoidal mass measuring $3 \times 5 \times 4 \mathrm{~cm}$ was visualised (Figure 1). Bronchoscopic biopsy of this mass revealed a benign papillomatous growth composed of squamous epithelial cells which were thrown into papillary projections (Figure 2). Fibrovascular cores showed mild chronic non-specific inflammatory cells. Malignant cells were not seen. Surgical removal of this papilloma was advised but the patient declined surgery. He, however, agreed upon regular follow-up visits. He was well at 2 months and at 6 months follow-up with no recurrence of hemoptysis. 


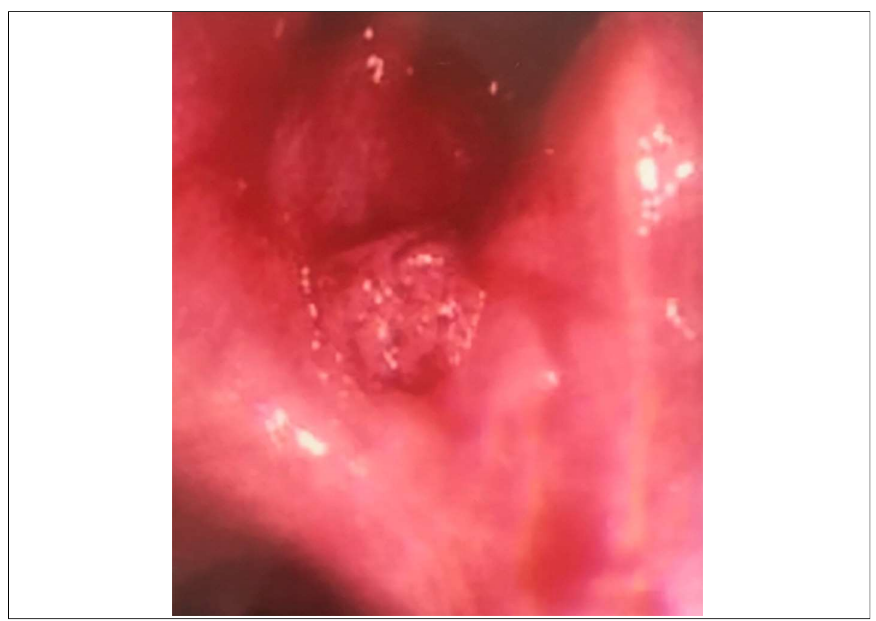

Figure 1: Bronchoscopy reveals a polypoid mass, measuring $3 \times 5 \times 4 \mathrm{~cm}$, located at the origin of right upper lobe bronchus. Note the characteristic soft friable appearance of the tumour.

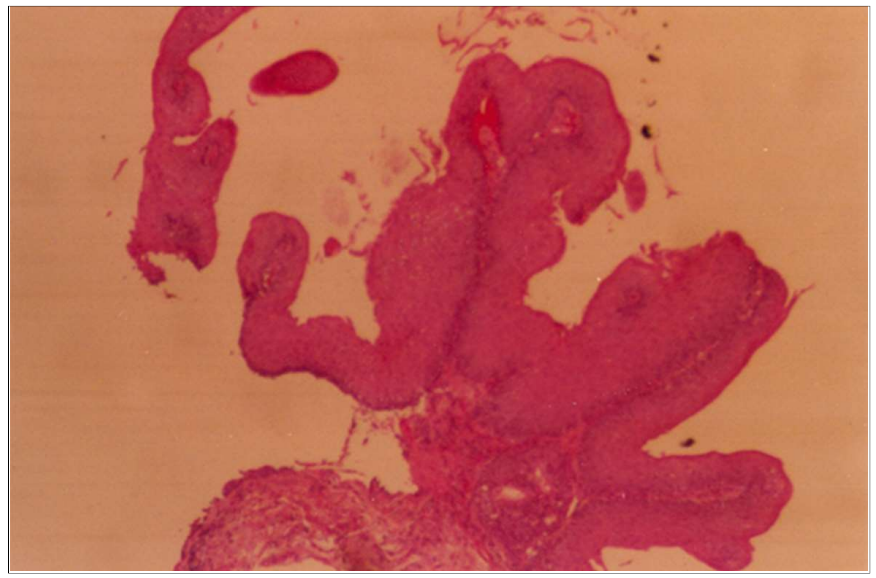

Figure 2: Low-power view showing a papillometous lesion with central fibrovascular cores (H\&E, x 4).

\section{DISCUSSION}

In developing areas of the world, such as the Indian subcontinent, tuberculosis is by far the most common cause of hemoptysis. ${ }^{2}$ Other frequent etiologies of hemoptysis are pneumonia, vasculitis, fungal infections, lung cancer, bronchitis, bronchiectasis and congenital heart disease. ${ }^{2}$ Because of their rarity, SEPs are generally not considered among the differentials. This makes their diagnosis challenging.

SEPs are very rare; only about a 100 have been reported in the medical literature. ${ }^{3}$ They occur with a higher frequency in men than women (3:1) and the vast majority are found in tobacco users. ${ }^{1}$ These tumours have been allocated three separate categories based on microscopic features: Squamous papilloma, glandular papilloma and mixed papilloma which displays a mixture of the other two types. ${ }^{1}$ Out of these, squamous papillomas have strongest association with tobacco smoking, and they are also most susceptible to malignant transformation. ${ }^{4}$ The patient had a solitary squamous papilloma of the bronchus.
These papillomas arise from the respiratory mucosal epithelium and measure between 0.7 and $2.5 \mathrm{~cm}$ in diameter. Grossly, they are soft polypoid lesions that are mostly exophytic, projecting into the airway lumen and narrowing it. This elevates the risk of post-obstructive infections and other complications such as atelectasis and bronchiectasis. ${ }^{5}$ SEPs may be completely asymptomatic incidental findings. 6 When symptoms are present, they may be cough, wheezing and asthma-like symptoms, hemoptysis and dyspnea. ${ }^{6}$ Obstructive symptoms may also be present. Many SEPs do not show up on chest X-ray, which can lead to a delay in the diagnosis. ${ }^{5} \mathrm{CT}$ is the preferred imaging modality and it usually shows a polypoidal nodule that may be obstructing the airway. ${ }^{5}$ The scan might show obstructive pneumonitis and atelectasis when there is complete airway obstruction. Partial but significant obstruction leads to reflex vasoconstriction resulting in diminished perfusion; this leads to hyperlucency of the affected areas. ${ }^{5}$ Distal airway and parenchymal involvement on CT manifests as numerous cavitate nodular lesions. 5 In our patient, the papilloma was discovered during bronchoscopy which was undertaken to identify the cause of his hemoptysis. The endoscopic findings depend upon the location and size of the lesion. Stenosis might be seen because of the SEP, but there has been a report of a papilloma that was situated distal to the site of stenosis. ${ }^{4}$ The lesions may or may not be pedunculated. Moreover, most reported SEPs have been described as having wart-like morphology. 4

Treatment options are chosen based on each individual case. The nonsurgical approach involves endoscopic excision of the lesion using snare electrocautery or yttrium aluminum garnet (YAG) laser.7,8 This is utilised because malignant spread to other areas of the respiratory tract may necessitate further removal procedures, so that as much pulmonary function can be saved as possible. ${ }^{8}$ The use of laser may render it impossible to conduct a thorough pathological inspection of the entire papilloma, which might have contained evidence of metaplastic change at the tumour base. 8 Snare electrocautery should, therefore, be the method of choice rather than YAG laser, because it allows for an examination of the complete papilloma for signs of malignancy. ${ }^{7}$ Surgery remains the most effective and most frequently employed method of treatment since it ensures removal of any occult malignancy and prevents recurrence that is sometimes seen after endoscopic excision owing to incomplete tumour resection. 7,8 During the surgical procedure, emphasis is placed on sparing as much lung tissue as possible in order to conserve the patient's pulmonary function. $7-9$ The choice of treatment the histologic subtype: surgical removal is the preferred approach for squamous papillomas on account of their known malignant potential. 1 On the other hand, for glandular papillomas (in which malignant transformation has never been reported), endoscopic treatment is 
recommended because it is less invasive. ${ }^{1}$ Ultimately, the choice of most suitable treatment hinges upon the location, size and histologic subtype of the lesion. It also dependson whether the lesion is recurring every few months to years after resection. Patient consent is also of paramount importance. Some patients, especially those in developing countries, may not have health insurance and lack the means to undergo any excision procedure. Such patiens are monitored carefully on a regular basis. Others might have financial means but are unwilling and apprehensive about surgery. As stated above, our patient also denied surgery because of this fear. Therefore, he was closely followed. When reviewed two months and six months later, he was stable and stated that there had been no further episodes of hemoptysis.

In a middle-aged to elderly smoker complaining of hemoptysis, a SEPs should be considered among the differentials, especially if the other more common causes have been ruled out. Moreover, in instances where a patient declines treatment, the papilloma needs to be monitored closely at regular intervals because of the risk of malignant transformation. Monitoring should also be continued for patients who have had the papilloma removed.

\section{PATIENT'S CONSENT:}

The authors obtained written informed consent and permission from the patient to submit this case report and the associated images for publication.

CONFLICT OF INTEREST:

Authors declared no conflict of interest.

AUTHORS' CONTRIBUTION: MSAM, MW, AHS, AS, JAK: Manuscript planning, literature search, writing, interpretion of available literature and derivation of relevant conclusions.

\section{REFERENCES}

1. Tryfon S, Dramba V, Zoglopitis F, lakovidis D, Sakkas L, Kontakiotis $\mathrm{T}$, et al. Solitary papillomas of the lower airways: Epidemiological, clinical, and therapeutic data during a 22-year period and review of the literature. J Thorac Oncol 2012; 7: 643-8.

2. Singh SK, Tiwari KK. Etiology of hemoptysis: A retrospective study from a tertiary care hospital from northern Madhya Pradesh, India. Indian J Tuberc 2016; 63:44-7.

3. Feng AN, Wu HY, Zhou Q, Sun Q, Fan XS, Zhang YF, et al. Solitary endobronchial papillomas with false impression of malignant transformation: Report of two cases and review of the literature. Int J Clin Exp Pathol 2015; 8:8607-12.

4. Paganin F, Prevot M, Noel JB, Frejeville M, Arvin-Berod C, Bourdin A. A solitary bronchial papilloma with unusual endoscopic presentation: Case study and literature review. BMC Pulm Med 2009; 9:40.

5. Stevic R, Milenkovic B. Tracheobronchial tumors. J Thorac Dis 2016; 8:3401-13.

6. Ghobain MA. Solitary endobronchial papilloma with malignant transformation and concomitant TB infection: Case report and literature review. Case Rep Pulmonol 2017; 2017:1606432.

7. Inoue $\mathrm{Y}$, Oka M, Ishii $\mathrm{H}$, Kimino $\mathrm{K}$, Kishikawa $\mathrm{M}$, Ito $\mathrm{M}$, et al. A solitary bronchial papilloma with malignant changes. Intern Med 2001; 40:56-60.

8. McNamee CJ, Lien D, Puttagunta L, Conlan AA. Solitary squamous papillomas of the bronchus: $A$ case report and literature review. J Thorac Cardiovasc Surg 2003; 126:861-3.

9. Himuro N, Niiya Y, Minakata T, Oshima Y, Kataoka D, Tazawa S, et al. A solitary bronchial squamous cell papilloma with increased 18-fluorodeoxyglucose uptake and high serum levels of squamous cell carcinoma antigen. J Thorac Dis 2018; 10:E435-E437.<smiles>C[14CH]C</smiles> 